International Journal of Bifurcation and Chaos, Vol. 12, No. 8 (2002) 1895-1907

(c) World Scientific Publishing Company

\title{
DEVELOPING CHAOS ON BASE OF TRAVELING WAVES IN A CHAIN OF COUPLED OSCILLATORS WITH PERIOD-DOUBLING. SYNCHRONIZATION AND HIERARCHY OF MULTISTABILITY FORMATION
}

\author{
A. SHABUNIN, V. ASTAKHOV and V. ANISHCHENKO \\ Physics Department, Saratov State University, Astrakhauskaya Str. 83, \\ Saratov, 410026, Russia
}

Received April 9, 2001; Revised September 14, 2001

\begin{abstract}
The work is devoted to the analysis of dynamics of traveling waves in a chain of self-oscillators with period-doubling route to chaos. As a model we use a ring of Chua's circuits symmetrically coupled via a resistor. We consider how complicated are temporal regimes with parameters changing influences on spatial structures in the chain. We demonstrate that spatial periodicity exists until transition to chaos through period-doubling and tori birth bifurcations of regular regimes. Temporal quasi-periodicity does not induce spatial quasi-periodicity in the ring. After transition to chaos exact spatial periodicity is changed by the spatial periodicity in the average. The periodic spatial structures in the chain are connected with synchronization of oscillations. For quantity researching of the synchronization we propose a measure of chaotic synchronization based on the coherence function and investigate the dependence of the level of synchronization on the strength of coupling and on the chaos developing in the system. We demonstrate that the spatial periodic structure is completely destroyed as a consequence of loss of coherence of oscillations on base frequencies.
\end{abstract}

Keywords: Chaos; chaotic synchronization; coupled oscillators; traveling waves.

\section{Introduction}

In recent years a great interests has been placed on problems of collective dynamics of simple oscillators which demonstrate different kinds of behavior from complete spatio-temporal synchronization to the formation of dissipative structures. These investigations have both theoretical and applied interests in different fields of physics [Kaneko, 1989a; Fisher, 1985] chemistry [Kuramoto, 1984] and biology [Mirollo \& Strogatz, 1990]. A series of works has been devoted to consideration of coupled maps arrays modeling different physical phenomena [Kaneko, 1989a, 1989b; Kuznetzov \& Kuznetzov, 1990]. Lattices of maps with chaotic behavior have rich dynamics and they allow to research the formation of regular and chaotic spatio-temporal structures resulting from synchronization of oscillations. However, the discrete time systems cannot demonstrate phase and frequency phenomena which we observe in real nature. Real oscillators demonstrate phase synchronization phenomenon for regular [Huygens, 1673] and chaotic [Rosenblum et al., 1996; Fujigaki et al., 1996] motions. As a result the existence of different phase structures is possible, when oscillations in subsystems have almost equal amplitudes and different phases and frequencies. These phenomena have been considered in a great 
number of works on phase oscillators. Most of the studies was devoted to the global mean-field coupling systems [Matthews \& Strogatz, 1990; Daido, 1996; Tass, 1997; Yeung \& Strogatz, 1999, 2000]. It was demonstrated that very simple periodic oscillators can demonstrate complex macroscopic behavior: periodic, quasi-periodic and even chaotic through quasi-periodic and period-doubling routes [Matthews et al., 1990]. The mean-field approach allowing to consider the behavior of the system as a whole, does not take into account local connections between elements, which can lead to the formation of local spatial structures. Locally coupled limitcycle oscillators was intensively investigated in the works by Daido [1997], Bressloff et al. [1997] and Bressloff and Coombes [1998]. Phase regularities in nearest-neighbor coupled oscillators have also been considered for circle maps [Pando, 2000].

It is known that chains of the simplest limitcycle oscillators with periodic boundary conditions exhibit traveling wave regimes when oscillations in neighboring sites differ for constant phase shifts. Daido considered a more complex oscillators chain (see [Daido, 1997]) and demonstrated that taking into account the second harmonics in the spectrum of oscillations can lead to novel behavior. Namely, he has found that temporal periodic oscillators can demonstrate spatially chaotic behavior. Hence, transition to more realistic models lead to new dynamics which cannot be realized in the simplest phase oscillators arrays. A number of works [Matias et al., 1997a; Matias et al., 1997b; Marino et al., 2000; Hu et al., 2000] have demonstrated that traveling wave regimes are possible for rings of chaotic oscillators (in the works [Matias et al., 1997a; Marino et al., 2000] they were called as "rotating waves"). Nevertheless, evolution of traveling waves occurring in complex systems with change in parameters has not been described in detail until now. What traveling wave regimes are possible in real chaotic oscillator chains? How do complicating temporal dynamics influence spatial structures? How do destroying of spatial structures connected with synchronization between nearestneighbor oscillators. In our work we focus on these questions. We have chosen a chain of perioddoubling self-oscillators with diffusion symmetric coupling. Similar systems have rich dynamics. They are characterized by developed phase multistability, when many regular and chaotic attractors coexist in phase space [Astakhov et al., 1989; Anishchenko et al., 1995]. They also demonstrate the possibility of the phenomena of full [Fujisaka \& Yamada, 1983] and partial [Inoue et al., 1998; Belykh \& Belykh, 2000] synchronization of chaos when all elements in the lattice or a part of such elements oscillate identically. A chaotic attractor related to the regime of synchronization is located inside the symmetric subspace $x_{1}=x_{2}=$ $\ldots x_{n}$, where $n$ is the number of all or a part of oscillators in the array. Destroying the synchronization is accompanied by the bubbling and riddled basins phenomena [Ashvin et al., 1994; Lai et al., 1996]. Bifurcational mechanisms of the synchronization loss and the multistability formation are found for several period-doubling bifurcations of every periodic orbit in the cascade of bifurcations [Astakhov et al., 1999]. These bifurcations lead both to the appearance of new stable attractors outside the symmetric subspace and to the appearance of regions of local transversal instability for the synchronous attractor [Astakhov et al., 1997a; Astakhov et al., 1998].

In Sec. 2 we consider the evolution of traveling waves with complicated dynamics during perioddoubling bifurcations. We build regions of stability for the waves with different spatial periods and demonstrate that short wavelength waves disappear with increasing coupling. Section 3 is devoted to a more detailed description of behavior of one family of regimes originating from a traveling wave with determined periodicity. We consider typical bifurcational transitions and build regions of different stable regimes on the parameter planes. In Sec. 4 we consider synchronization of oscillations. We demonstrate that the complete synchronization breaks after transition to chaos. After these oscillators are nearly synchronized, to appreciate the quantity measure of their synchronization we use the averaged coherence function approach [Anishchenko et al., 2000]. The proposed measure makes clear physical sense: it shows what part of the full power relates to coherent oscillations. Using this characteristic we demonstrate that the traveling wave regimes are destroyed gradually with decreasing coupling and this process is accompanied by decreasing mutual coherence functions and therefore by decreasing the levels of synchronization. The complete destruction of the average space-periodicity is connected with the loss of the coherence on the main frequencies in the spectrum. The conclusion contains the summary of the obtained results. 


\section{The Evolution of Traveling Waves with Parameters Changing}

As a model we used a chain of 30 Chua's self-oscillators coupled via a resistor:

$$
\begin{aligned}
& \dot{x}_{i}=\alpha\left(y_{i}-x_{i}-f\left(x_{i}\right)\right) \\
& \dot{y}_{i}=x_{i}-y_{i}+z_{i}+\gamma\left(y_{i-1}+y_{i+1}-2 y_{i}\right) \\
& \dot{z}_{i}=-\beta y_{i}
\end{aligned}
$$

(where

$$
f(x)= \begin{cases}b x+a-b & \text { if } x>1 \\ a x & \text { if }|x| \leq 1 \\ b x-a+b & \text { if } x<-1\end{cases}
$$

$i=1,2, \ldots, N ; N=30$ ) with periodic boundary conditions:

$$
x_{1}=x_{N}, \quad y_{1}=y_{N}, \quad z_{1}=z_{N} .
$$

All oscillators are identical. The behavior of a single oscillator is widely described in the literature (see e.g. [Komuro et al., 1991]). It is characterized by period-doubling bifurcations cascade and bistability, when two symmetric attractors formed near two nontrivial equilibria $P_{1}$ and $P_{2}$ coexist in the phase space. With parameter $\alpha$ increasing these attractors merge and as a result double scroll chaotic attractor appears. The system of two coupled oscillators behave in a more complex way [Anishchenko et al., 1995]. It demonstrates both period-doubling bifurcations cascade and tori breaking routes to chaos. The system is characterized by the developed multistability and the complete synchronization of chaos phenomenon. The in-phase complete synchronization of chaos exists at sufficiently large coupling. With decreasing coupling the regime of synchronization breaks (see [Anishchenko et al., 2000]). This process is accompanied by the bubbling of attractor and riddled basins phenomena. Arrays and lattices of Chua's oscillators with different types of coupling have been investigated in a series of works (see e.g. [Perez-Munuzuri et al., 1993; Alexeev et al., 1995; Matias et al., 1997a; Marino et al., 2000]).

We investigated the system (1) by means of computer simulations. The parameter $\alpha$ and the coefficient of coupling $\gamma$ were controlling parameters. Other parameters were fixed at values: $a=$ $-8 / 7, b=-5 / 7, \beta=-22$. (a) $\mathrm{N}=1$

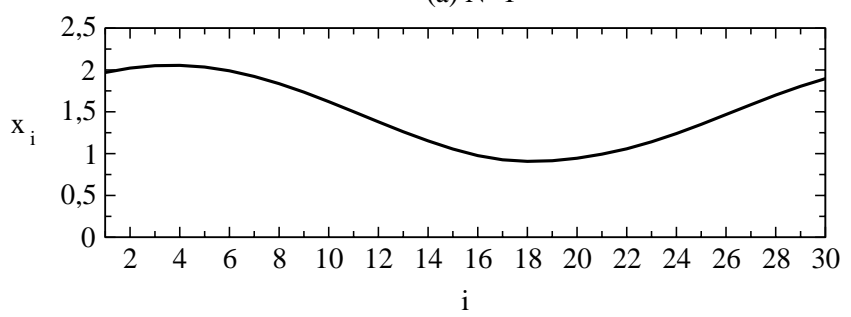

(b) $\mathrm{N}=2$

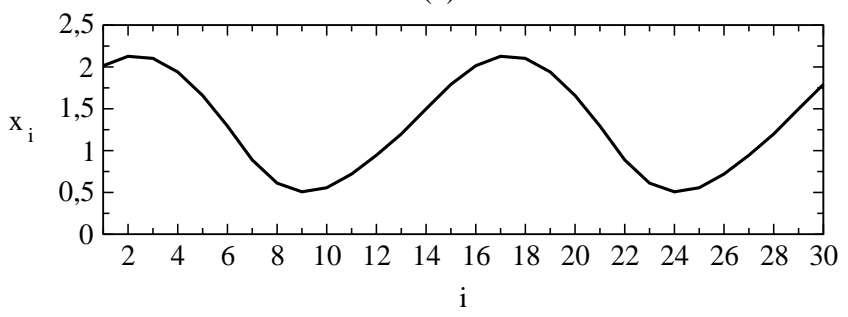

(c) $\mathrm{N}=3$

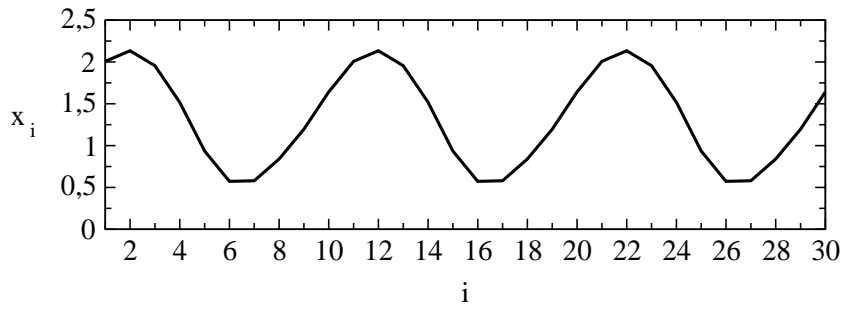

(d) $\mathrm{N}=5$

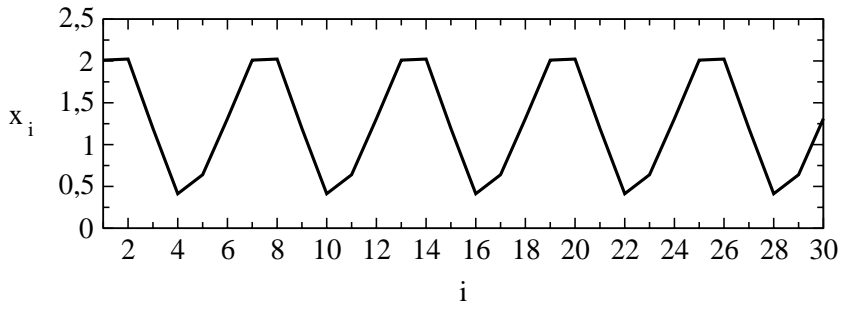

Fig. 1. Spatial structures of simplest traveling waves with different wavelengths.

Until $\alpha<8.78$ there are no oscillations in the system. At $\alpha \geq 8.78$ nearly harmonic period-one limit-cycle oscillation regimes with different spatial periods can be found depending on the value of the coupling and on the initial values. They are characterized by equal amplitude and shifted phases of oscillations in every site in the chain. These regimes are traveling waves rotating along the ring with constant phase velocity. For visualization of the spatial structure of the traveling waves we used the Poincaré section. We fixed the value of the $x_{i}$ variable of every oscillator while the variable $y_{1}$ crosses through the zero level from positive to negative values. The chosen simplest waves with different wavelengths are presented in the Fig. 1. The abscissa axis in Fig. 1 marks the location of oscillator in 
the chain, the ordinate axis denotes the value of $x_{i}$ at the Poincaré section. If all oscillators move in-phase we can see a straight horizontal line. If there are some phase shifts between oscillations we see different periodic curves. Because of the spaceperiodicity of the chain the summary phase shift along the entire chain must be proportional to $2 \pi n$. Figure 1 presents states with $n=1$ [Fig. 1(a)], $n=2$ [Fig. 1(b)], $n=3$ [Fig. 1(c)] and $n=5$ [Fig. 1(d)]. They correspond to phase shifts between oscillations of neighboring oscillators of $\pi / 15$, $2 \pi / 15, \pi / 5$, and $\pi / 3$, respectively. It is clear that for chains with finite numbers of elements only finite number of spatially periodic phase waves are possible because the length of the chain must be divisible by the spatial period. In our case other possible states remain with $n=6$ and $n=15$. The latter case is related to the complete antiphase synchronization. Traveling waves with these spatial periods ( $\Lambda=5$ and $\Lambda=2$ oscillators) were not found in the considered chain, maybe because of very narrow regions of stability.

Bifurcations of the states represented in Fig. 1 which take place with the increasing of parameter $\alpha$ lead to complicating temporal dynamics in every oscillator. These bifurcations are the period-doubling and tori birth bifurcations of regular attractors, and bound-merging bifurcations of chaotic attractors. A (a) $\alpha=1.14, \gamma=0.02$

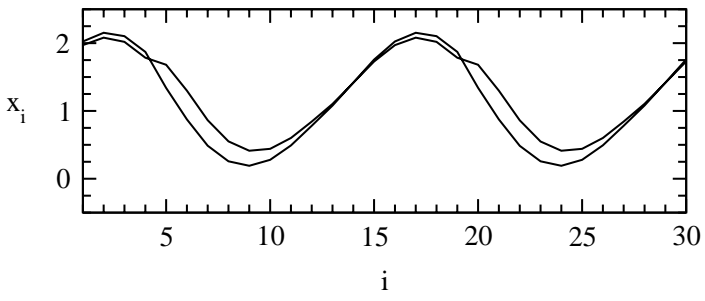

(b) $\alpha=11.48, \gamma=0.02$

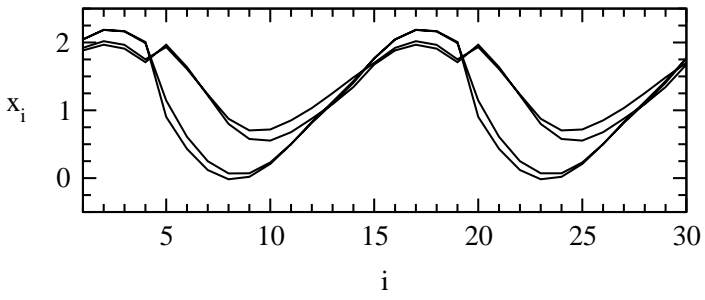

(c) $\alpha=11.54, \gamma=0.1$

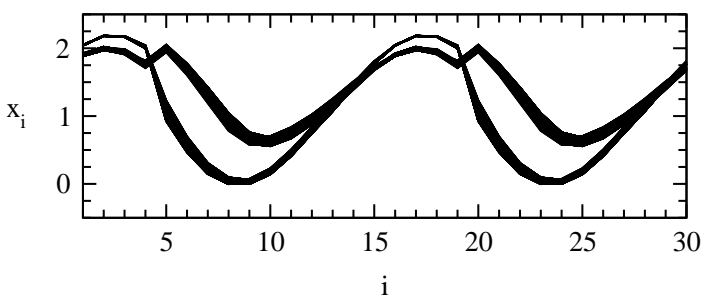

(d) $\alpha=11.4, \gamma=0.2$

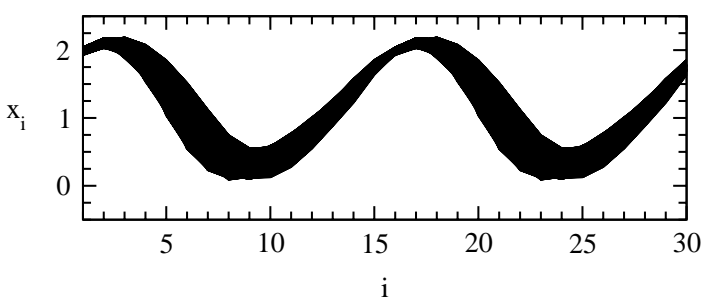

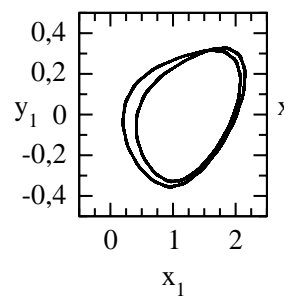
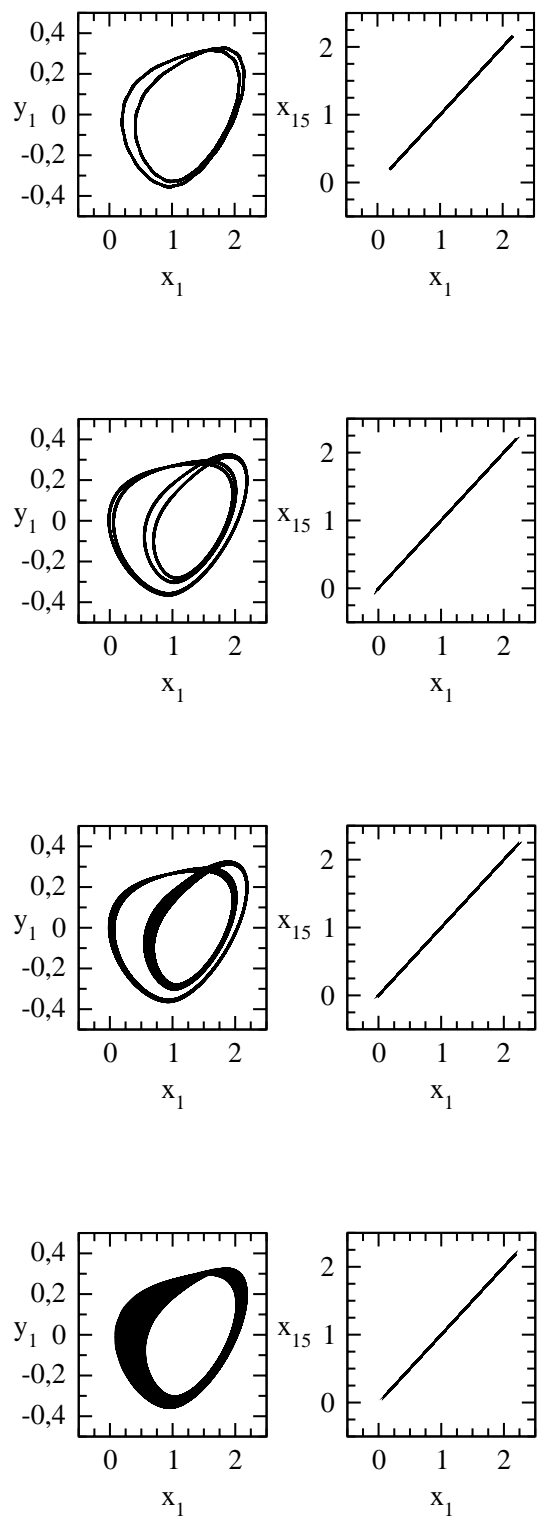

Fig. 2. Developing of the regular traveling waves with the parameter $\alpha$ increasing. 
more detailed structure of the plane of the regimes will be described in Sec. 3. Figure 2 presents the complications of regular traveling waves with parameter $\alpha$ increasing. It describes the evolution of regimes based on the initial state with spatial period $\Lambda=N / 2$ [Fig. 1(b)]: the traveling wave with a period-two cycle $2 C_{N / 2}$ temporal behavior [Fig. 2(a)], with a period-four cycle $4 C_{N / 2}$ behavior [Fig. 2(b)], a two-band torus $2 T_{N / 2}$ [Fig. 2(c)] and a one-band torus $1 T_{N / 2}$ [Fig. $\left.2(\mathrm{~d})\right]$ behavior. In this figure we have built the spatial structures and projections of phase portraits to the planes $x_{1}-y_{1}$ and $x_{1}-x_{16}$. The latter projection demonstrates that the mentioned regular regimes have exact spatial periodicity $x_{i}=x_{i+15}$. We see that increase of the temporal period does not lead to changing of the spatial period and temporal quasi-periodicity does not lead to spatial quasi-periodicity. The development of temporal regimes take place on a background of the original spatial periodic

(a) $\alpha=11.57, \gamma=0.05$
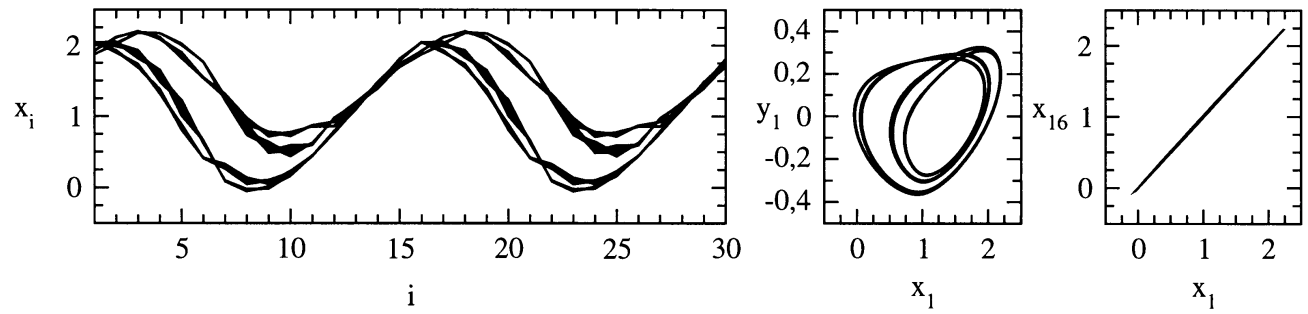

(b) $\alpha=11.61, \gamma=0.05$
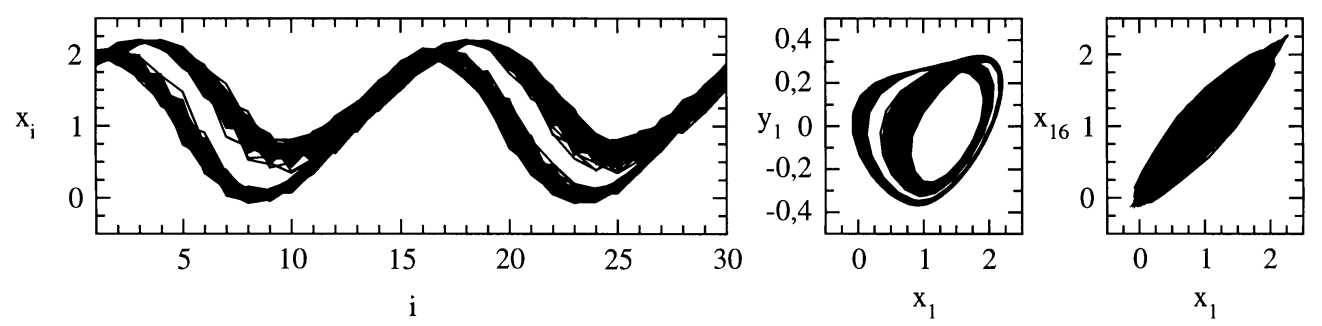

(c) $\alpha=11.66, \gamma=0.05$
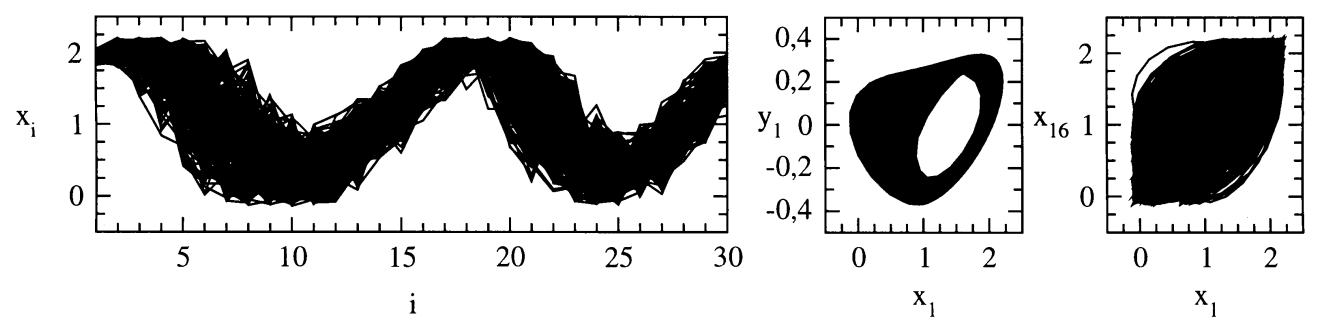

(d) $\alpha=11.79, \gamma=0.05$
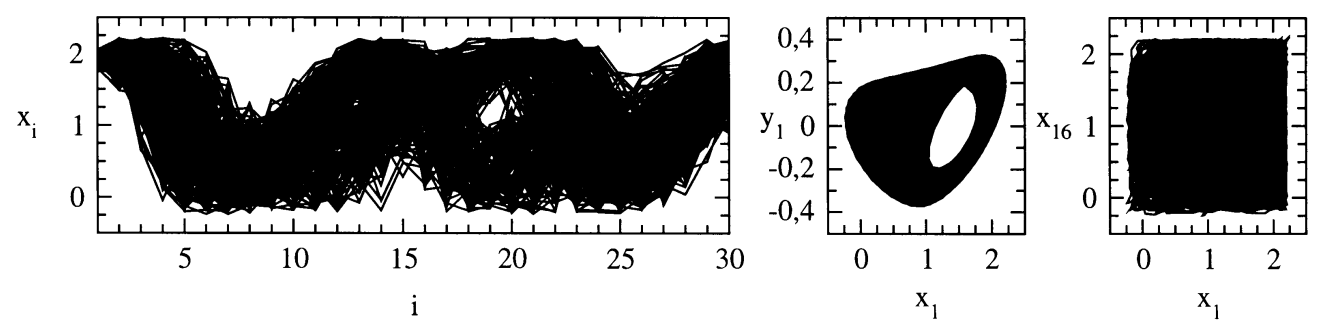

Fig. 3. Developing of the chaotic traveling waves with the parameter $\alpha$ increasing. 
structure that is destroyed only after transition to chaos. The evolution of chaotic regimes is presented in Fig. 3. This figure presents the cases of a four-band attractor [Fig. 3(a)], a two-band attractor [Fig. 3(b)] and one-band attractor Figs. 3(c) and $3(\mathrm{~d})$. The chaotic regimes do not remain exactly spatio-periodic. It can be seen from the "mutual" projection $x_{1}-x_{16}$ which is not a line more, but has finite "thickness". For many-band chaotic attractors it is very thin, i.e. the regimes are almost exactly space-periodic [Fig. 3(a)]. With the development of chaos the projection becomes more "thick" that means that the regime loses spatio-periodicity. In the regime of developed one-bound chaotic attractor the mutual projection looks like a square [Fig. 3(c)]. The mutual projection of the phase portraits and the spatial diagrams demonstrate that chaotic oscillations are not space-periodic more. However, as it will be demonstrated later, they remain space-periodic on an average.

Stability of traveling waves depends on their wavelength and on the coupling strength. Increasing the coupling coefficient $\gamma$ leads to loss of stability for short wavelength modes and as a result of transition to more long wavelength ones. Figure 4 presents regions of stability for the families of regimes originating from the traveling waves described in Fig. 1. The upper boundaries (symbols) mark destroying of the spatial structure due to

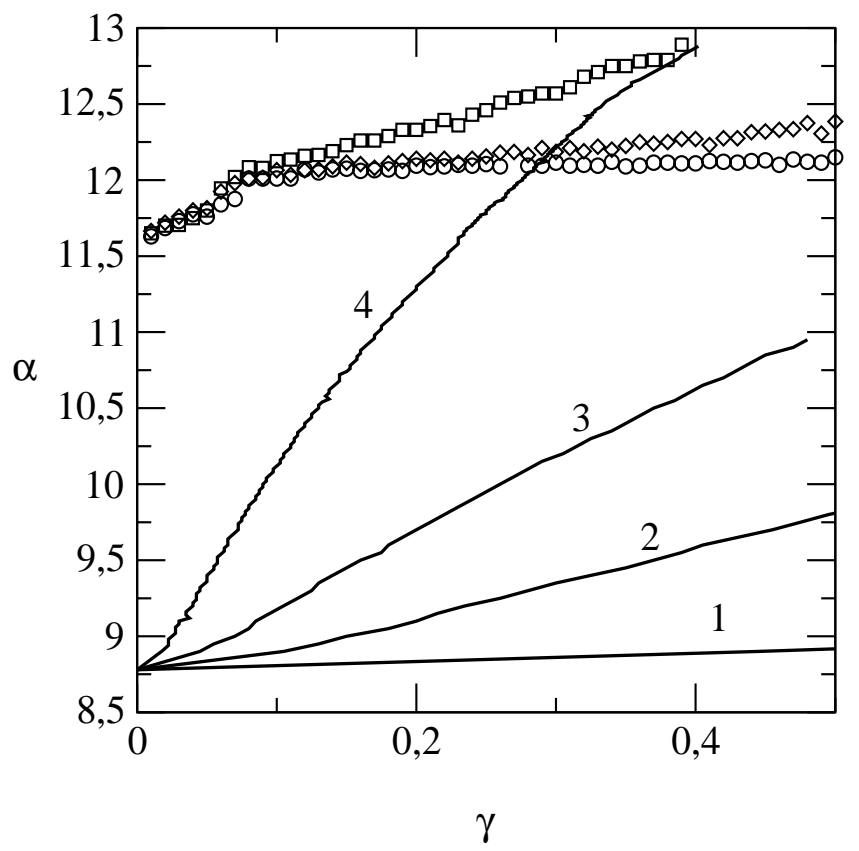

Fig. 4. Regions of stability for the families of waves with different wavelengths. developing chaos. The right boundaries (lines) mark the loss of stability in a sudden way. As a result of it the system transits to regimes with longer wavelengths. The line "1" bounds a family of regimes with spatial period $N$ [see Fig. 1(a)]. This line is almost horizontal. From the right side of this line only spatially-homogeneous regime is possible. The lines "2", "3", "4" are boundaries of families of regimes with spatial periods $N / 2, N / 3, N / 5$, respectively. From the left-hand sides of these lines waves with these spatial structures are observed. On crossing these lines only stable waves with longer wavelengths can exist. Namely, from the left side of line " 4 " the families of regimes with wavelengths $N / 5, N / 3, N / 2, N$ and spatio-homogeneous regime coexist. From the left side of the line " 3 " - with wavelengths $N / 3, N / 2, N$ and spatiohomogeneous, from the left side of the line " 2 " with wavelengths $N / 2, N$ and spatio-homogeneous regimes coexist. The spatio-homogeneous regimes exist at any coupling. We see that stability of the traveling waves depend on coupling. The increasing of coupling leads to loss of stability for regimes with shorter spatial periods.

Unfortunately, the large dimension of the considered system does not lead to the possibility to carry out a detailed bifurcational analysis. Taking into account the location of bifurcational lines $1-4$ and bifurcations in the coupled oscillators with smaller dimensions [Anishchenko et al., 1995; Astakhov et al., 1997b] we can propose a hypothesis that the mentioned spatially periodic regimes originated as a result of bifurcations of the same equilibrium $P_{1,2}$. In our opinion the bifurcational mechanism can be the following. If we have a single oscillator, the periodic period-one cycle appears through the Hopf bifurcations of the equilibria $P_{1}$ or $P_{2}$. After this, a stable period-one cycle appears. In the system of two coupled oscillators [Astakhov et al., 1997b] the equilibrium undergoes two consistent Hopf bifurcations. As a result of the first bifurcation the stable in-phase (or antiphase, depending on the type of coupling) periodone cycle appears from the stable equilibrium point and the point becomes saddle. After the second Hopf bifurcation the saddle equilibrium becomes unstable along another direction and the saddle antiphase (or in-phase) period-one cycle appears. Then, with increasing of the parameter $\alpha$ the saddle cycle can become stable through the pitchfork (for example) bifurcation. If we have many-oscillators system the equilibrium point can undergo several 
Hopf bifurcations. The first Hopf bifurcation leads to appearance of period-one spatio-homogeneous stable regime. After this the equilibrium becomes a saddle. The cycles that satisfy the spatioperiodicity conditions appears as a result of the following Hopf bifurcations. They are saddle. The regimes with longer spatial periods appear earlier than the short wavelength ones. Then, with the parameter $\alpha$ increasing these saddle cycles become stable. In the framework of the considered hypothesis the lines 1-4 are bifurcational lines with a bifurcational condition for a multiplier of the correspondent cycle: $\mu=+1$.

\section{Typical Bifurcational Transitions and Structure of the Parameters Plane for a Family of Spatio-Periodic Regimes}

In this section we carry out a more detailed analysis of traveling waves formed on the base of the period-one cycle regime with $\Lambda=N / 2$ [Fig. 1(b)]. The common structure of the regimes on the parameters plane $\gamma-\alpha$ is presented in Fig. 5. The region of stability of this family of regimes is bounded by lines " 1 ", " 5 " and " 6 ". The line " 1 " bounds this region from the right. On crossing the line the regimes with wavelength $\Lambda=N / 2$ loses their stability in a sudden way and the system transits to waves with larger wavelengths. Over this line and before the line " 2 " the stable period-one cycle is observed. On the line " 2 " the system transits to quasi-periodic behavior. This is the line of the torus $2 T_{N / 2}$ [Fig. 2(d)] birth. For large coupling the system evolves at the base of this torus and demonstrates transition to chaos through the torus breaking (dashed line " 4 " in Fig. 5). Over this line a one-band chaotic attractor exists. For small coupling the transition to chaos occurs through the period-doubling bifurcations and quasi-periodic behavior originates from cycles with double periods. This region of smaller coupling is bounded by the line " 3 ". Over this line the period-two cycle is observed [Fig. 2(a)] which transits to the two-torus $2 T_{N / 2}$ on the line " 5 " [Fig. 2(c)]. Then on the base of this torus there are two possibilities: at larger coupling the torus breaks with the appearance of two-band chaotic attractor, and for smaller coupling (on the line "6") it transits to a period-four cycle [Fig. 2(b)] which then undergoes tori birth bifurcation. The picture of regimes and bifurcational lines in Fig. 5 is rather robust. We restrict ourselves to only several first

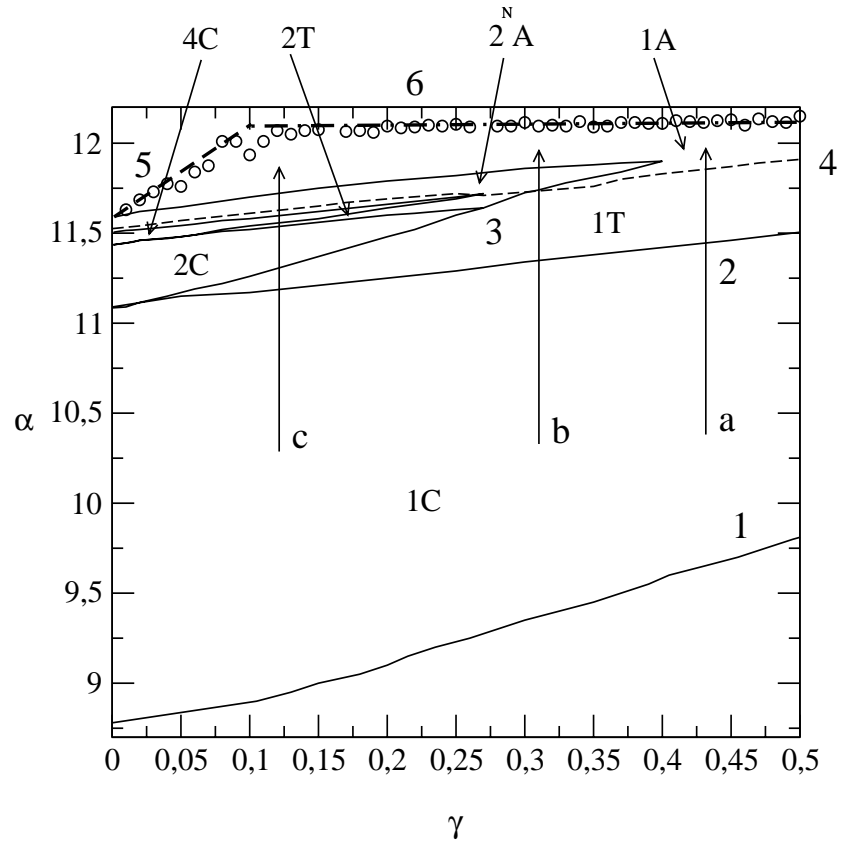

Fig. 5. Diagram of regular and chaotic regimes originating from the wave with spatial period $N / 2$.

period-doubling and tori birth bifurcations, up to period eight. The analysis holds, and comparing with the analysis of typical bifurcations in systems of two coupled via resistor [Anishchenko et al., 1995] and via capacity [Astakhov et al., 1997b] oscillators present typical scenarium for evolutions of regimes on the base of the space-periodic period-one cycle. The route to chaos goes through several perioddoubling and tori birth bifurcations. The number of bifurcations decreases with increasing coupling. At large coupling we have a chain of two bifurcations: $1 C \rightarrow 1 T \rightarrow 1 A$ (the arrow $a$ in Fig. 5), at smaller coupling there is a chain of five bifurcations: $1 C \rightarrow 1 T \rightarrow 2 C \rightarrow 2 T \rightarrow 2 A \rightarrow 1 A$ (the arrow $b$ in Fig. 5), at even smaller coupling $1 C \rightarrow 1 T \rightarrow$ $2 C \rightarrow 2 T \rightarrow 4 C \rightarrow 4 T \rightarrow 4 A \rightarrow 2 A \rightarrow 1 A$ (the arrow $c$ in Fig. 5). The described dependence of the bifurcation chains on coupling suggest the common bifurcational mechanisms for the changing of temporal behavior of the family of spatio-periodic regimes: At finite coupling we have a finite chain of period-doubling and tori birth bifurcations which lead to chaos through the final tori breaking bifurcation. The length of the chain increases with decreasing coupling and at the limit of zero coupling tends to infinity.

The structures of the phase-space and typical bifurcations are similar for spatio-periodic regimes with other wavelengths. 


\section{Synchronization of Chaos in the Chain of Oscillators}

The considered complex spatial structures of oscillations in the chain are results of synchronization. Usually the term "complete synchronization" is used for the behavior when all oscillations in the chain are equal in every moment of time $\left(x_{i}(t)=\right.$ $x_{k}(t)$ for all $i$ and $k$ ) namely, when the attractor belongs to the symmetric subspace $x_{1}=x_{2}=\ldots x_{N}$ [Heagy et al., 1994]. The dimension of the subspace is equal to the dimension of the single oscillator. The case when oscillations are equal up to time-delay: $x_{i}(t)=x_{i+1}(t+\tau)$ was called as "lag synchronization" [Rosenblum et al., 1997]. From another point of view the case when all oscillators can be divided into several groups in which every one of the oscillations are equal was called partial synchronization [Belykh \& Belykh, 2000]. Therefore, the case of considered spatio-periodic structures can be called as both lag-synchronization and as partial synchronization. There is a more general approach to the chaotic synchronization definition which can be applicable to different cases of mutual behavior. This is the "generalized synchronization" [Rulkov et al., 1995]. In the framework of this approach we shall call oscillations as fully synchronized if there is deterministic connection between states of any oscillators in the chain: $x_{i}(t)=f\left(x_{k}(t-\tau)\right)$. This is the case of full synchronization. When this equality is fulfilled not exactly, or it takes place from time to time (for example in the case of bubbling phenomenon) we have partially synchronized oscillations. In this case it is necessary to have quantity measure of this synchronization, which will demonstrate changing of synchronization level from 0 for unsynchronized oscillators till 1 for fully synchronized ones. This measure must make clear the physical sense, and must be easily calculated by standard algorithms using time-series from coupled oscillators and it must be universal, namely can be applicable to different cases of synchronization. We have proposed such synchronization measure in the work [Anishchenko et al., 2000] where it has been used for investigation of complete synchronization loss and bubbling phenomenon in the system of two coupled chaotic self-oscillators. This measure is constructed on the base of the coherence function:

$$
\sigma(\omega)=\left|\left\langle\frac{\chi_{x_{1}}(\omega) \chi_{x_{2}}^{*}(\omega)}{\left|\chi_{x_{1}}(\omega) \| \chi_{x_{2}}(\omega)\right|}\right\rangle\right|
$$

where $\chi_{x_{i}}$ is spectral density on the time-series of the $i$ th oscillator, $\omega$ is a frequency, $\langle\cdots\rangle$ denotes averaging on an ensemble of realizations. The coherence function is equal to 1 for the frequencies where oscillations are coherent and equal to 0 where they are independent. To evaluate the level of synchronization on all frequencies it is necessary to average the coherence functions with weight function. The weight function must take into account what part of the entire power belongs to the corresponding spectral terms. The complete formula for calculating the synchronization level has the form:

$$
S=\frac{\int_{0}^{\infty} \sigma(\omega)\left(\left\langle\left|\chi_{x_{1}}(\omega)\right|^{2}\right\rangle+\left\langle\left|\chi_{x_{2}}(\omega)\right|^{2}\right\rangle\right) d \omega}{\int_{0}^{\infty}\left(\left\langle\left|\chi_{x_{1}}(\omega)\right|^{2}\right\rangle+\left\langle\left|\chi_{x_{2}}(\omega)\right|^{2}\right\rangle\right) d \omega}
$$

Using the formula (3) we can interpret the value $S$ as a part of the power which relates to coherent motions i.e. the motions on the frequencies where the phases are locked. The proposed measure is similar to the "order parameter" which was used for the description of measure of collective synchronizations in ensembles of phase oscillators [Kuramoto \& Nishikava, 1987; Matthews \& Strogatz, 1990], but it is applicable for more complex regular and chaotic oscillators. Phase coherence was used as a chaotic synchronization measure in the work [Mormann et al., 2000] where authors used instantaneous phase approach. In our opinion the coherence function approach is more universal because it is applicable also for the cases when instantaneous phase cannot be defined.

Let us consider the family of traveling waves with wavelength $N / 2$ [Fig. 1(b)]. The boundary of the family of regimes with this space-periodicity consists of three segments. The first one is the line "1" [Fig. 5]. On this line the regimes lose their stability in a sudden way and the system transits to regimes with longer wavelengths. From the left side of this line and until lines " 6 " and " 7 " there are the exactly space-periodic and nearly space-periodic regimes with this wavelength. The exact spaceperiodicity is destroyed at once after transition to chaos. In the chaotic region there is no exact spaceperiodicity, but it is observed "in the average". Figure 6 demonstrates averaged spatio-temporal diagrams with decreasing coupling. In this figure we observe gradual destruction of the averaged spaceperiodic structure. The corresponding temporal behavior is in a one-band chaotic attractor. For large 
(a) $\alpha=11.78 \gamma=0.15$

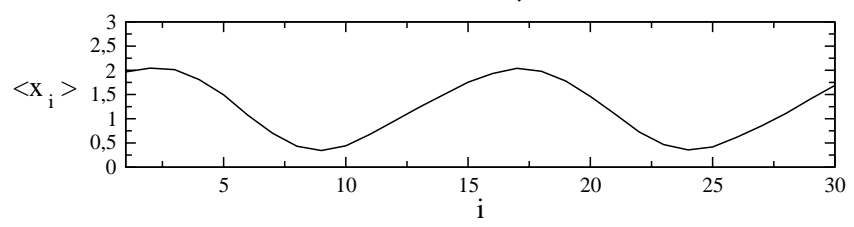

(b) $\alpha=11.78 \gamma=0.045$

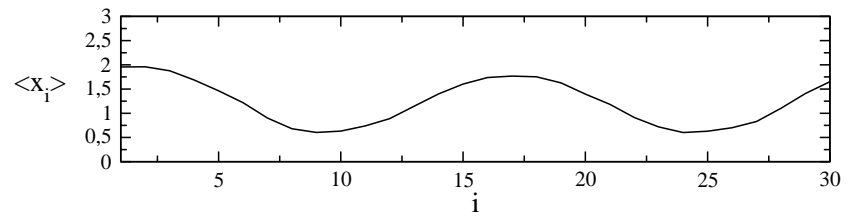

(c) $\alpha=11.78 \gamma=0.035$

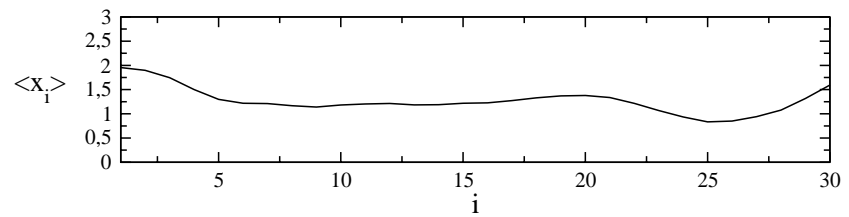

(d) $\alpha=11.78 \gamma=0.03$

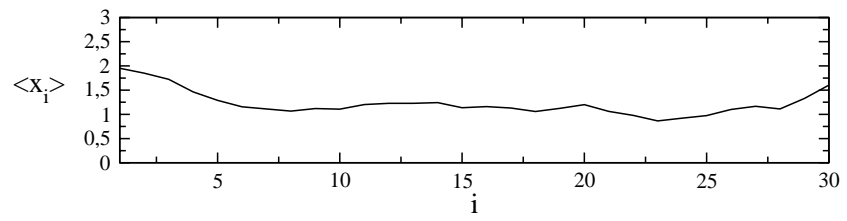

(e) $\alpha=11.78 \gamma=0.01$

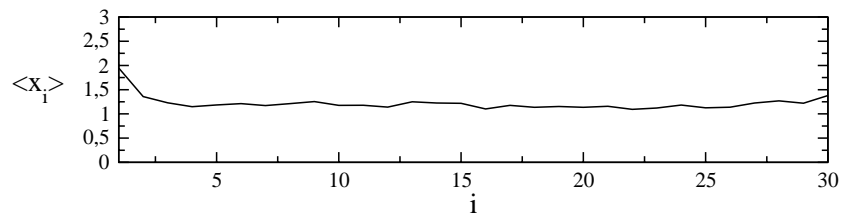

Fig. 6. The averaged spatial structure of the wave destroyed with developing temporal chaos.

coupling the averaged spatial diagram looks like the original traveling regime [compare Figs. 1(a) and 5(a)]. Then, at $\gamma<0.045$ it begins to change its form. At $\gamma=0.035$ the form of the spatial picture becomes more flat, though it preserves the original structures with two maximums [Fig. 5(c)]. For smaller coupling it completely "forgets" the previous structure. Due to the gradual nature in processing the destruction of space-periodicity, the line " 6 " is not a bifurcational line, but it characterizes a determined step in this process. Namely, the points of this line (they are marked by "o") denotes the minimum coupling behind which the spatial picture becomes qualitatively different. The gradual destroying of averaged space-periodicity is observed only for developed temporal chaos. If we choose the parameter $\alpha$ correspondent to two-band (or manyband) chaotic attractor the averaged space-periodic structure is preserved until zero coupling. From the top the region of the averaged space-periodicity is bounded by line "7". Increasing of the parameter $\alpha$ leads to developing of temporal chaos and to destroying of space-periodic structure. For middle coupling values it is destroyed gradually like in the case of decreasing coupling [see Figs. 6(a)-6(c)]. For strong coupling the averaging periodic structure is preserved until the transition to the double-scroll regime [Fig. 6(d)].

Let us look on the process of destroying spatial periodic structure from the point of view of mutual synchronization of oscillators. In the regular region, until transition to chaos we have the case of full synchronization. These regimes are characterized by exact equalities $x_{i}(t)=x_{i+N / 2}(t)$ and $x_{i}(t)=x_{i+1}\left(t+\tau_{i}\right)$ for every oscillator (see Fig. 2). The synchronization level $S$ is exactly equal to 1 . With the increasing of parameter $\alpha$ when

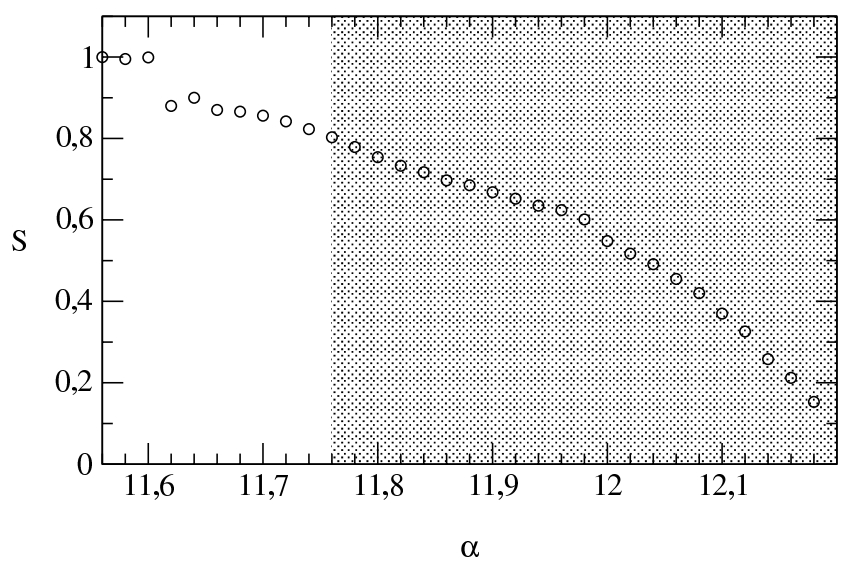

(a)

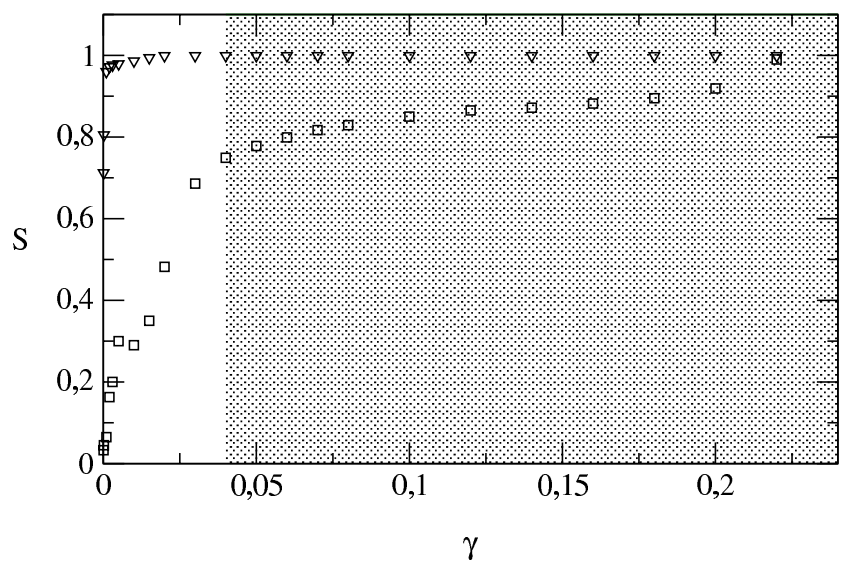

(b)

Fig. 7. Dependence of the level of chaotic synchronization on (a) developing temporal chaos and (b) decreasing coupling. 
oscillations become chaotic the equality $x_{i}(t)=$ $x_{i+N / 2}(t)$ is not fulfilled exactly. This leads to "thickness" of the mutual projection of the phase portrait (Fig. 3). The synchronization level becomes smaller than 1. After the transition to chaos it is near 0.99 (at $\alpha=11.57$ ), then with further chaotization it becomes smaller: 0.77 at $\alpha=11.79$ [Figs. $4(\mathrm{a})-4(\mathrm{e}), 0.56$ at $\alpha=12.0$ and 0.13 at $\alpha=12.25$ (for double-scroll regime). The dependence of the synchronization level $S$ on the parameter $\alpha$ is built in Fig. 7(a). Figure 7(b) demonstrates decreasing of the synchronization level with decreasing coupling. The gray color marks the regions where the averaged periodic structure is destroyed. It relates to the level of synchronization less than approximately 0.8 .

The synchronization level depends on the distance between interacting oscillators in the chain. We have built this dependence for the cases of twoband chaotic attractor, one-band attractor and for a developed temporal chaos with destroyed spatial

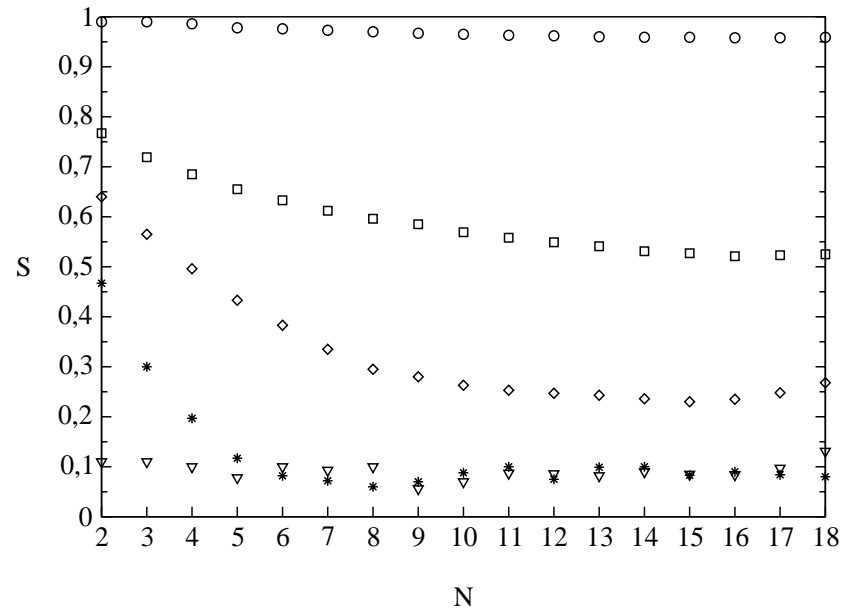

Fig. 8. Dependence of the level of synchronization on the distance between oscillators in the chain.

structure (Fig. 8). For two-band attractor (marked by "०") we see almost full synchronization $(S \approx 1)$ which is gradually decreased with increasing of distance. The developed temporal chaos with almost (a) $\alpha=11.57 \gamma=0.05$
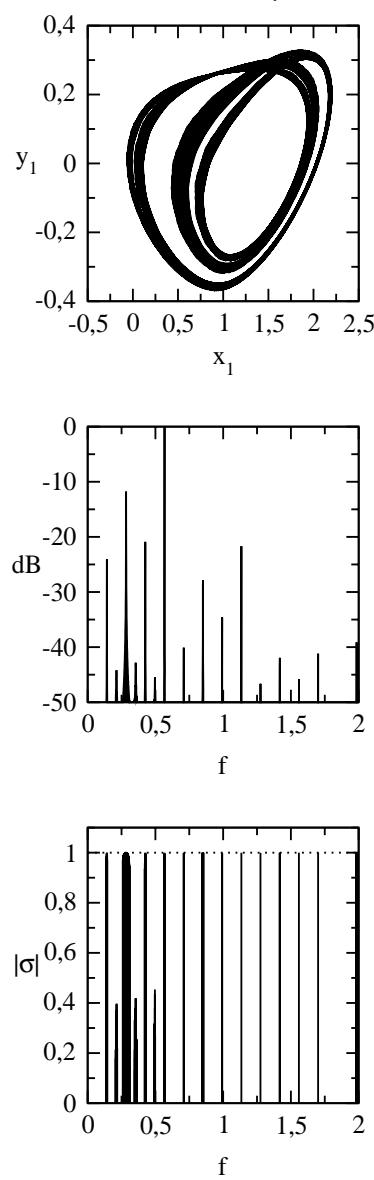

(b) $\alpha=11.61 \gamma=0.05$
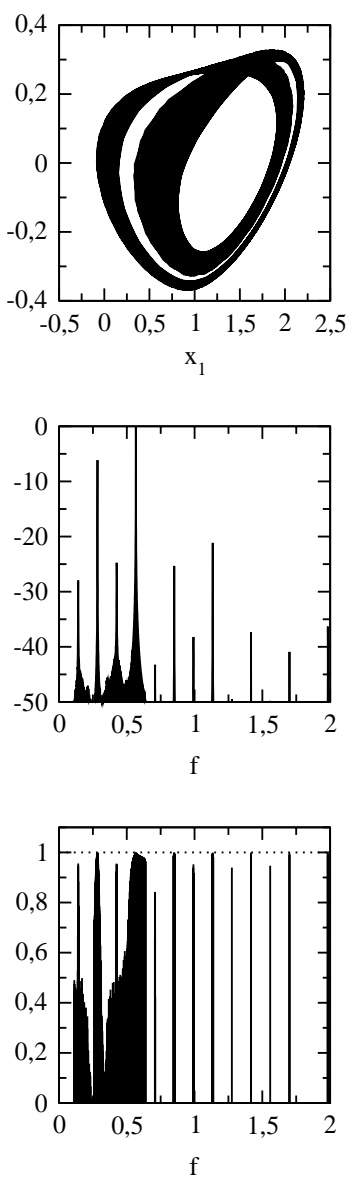

(c) $\alpha=11.79 \gamma=0.05$
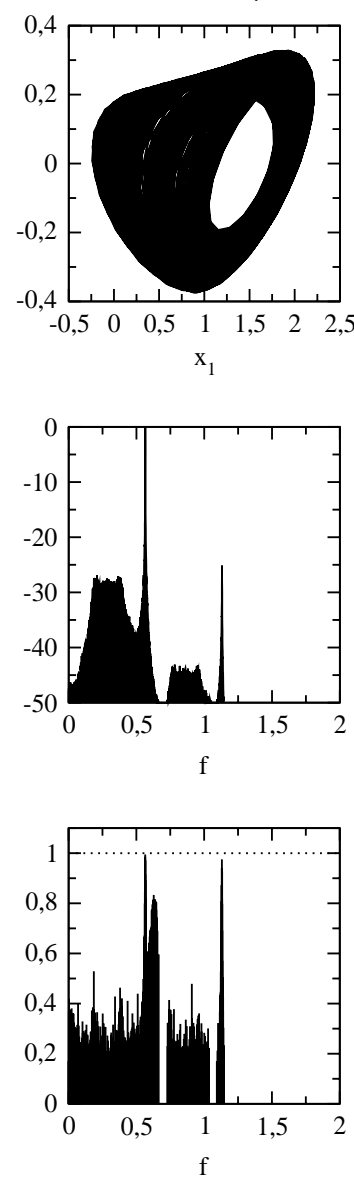

Fig. 9. Changing in the power spectrum of oscillations and of the module of the coherence function with developing of temporal chaos. 
(a)

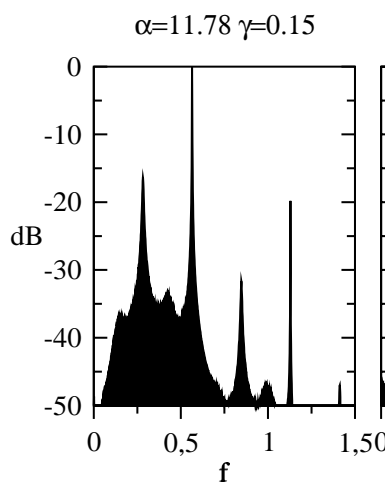

(b)

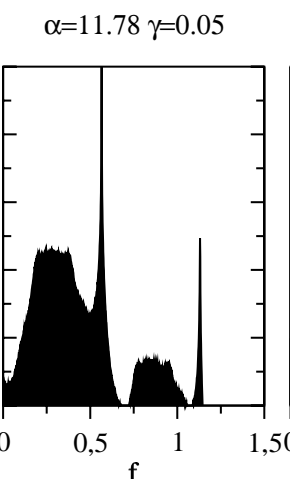

(c)

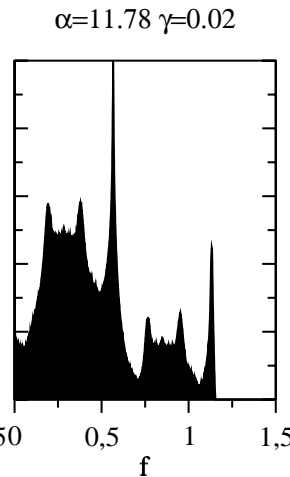

(d)
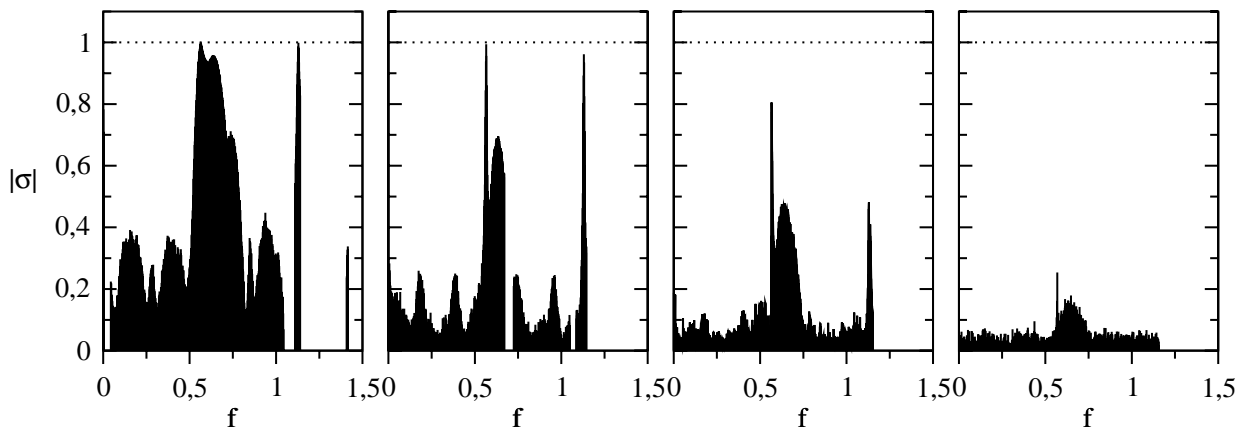

Fig. 10. Losing of the coherence between dynamics of the neighbor oscillators with the decrease in coupling.

periodic spatial structure is characterized by decreased level of synchronization $(S \approx 0.8)$ which decreases rapidly with distance. When the spatial structure is fully destroyed the level of synchronization very quickly falls to almost zero level (in our case it is a value of several percents, the precision of the experiment). At $\gamma=0.02$ ("**) the distance at which the level of synchronization falls from $S \approx 0.4$ till zero is five oscillators. At smaller coupling $(\gamma=0.002)$ the level of synchronization falls to zero at distance of one oscillator.

Figure 9 demonstrates rebuilding of the power spectrum and the coherence function with chaotization of oscillations. The coherence functions is built for oscillations of nearest-neighbor oscillators. Figure 9 (a) corresponds to a four-band attractor. The coherence function is equal to 1 for peaks on harmonics $2 \pi / T$ and first sub-harmonics $2 \pi / 2 T$ of spectra and has smaller values for remaining frequencies. Figures 9(b) and 9(c) correspond to twoand one-band attractors. It is seen that the coherence is equal to 1 for main peaks and it remains very close to 1 for first sub-harmonics [Fig. 9(b)]. The coherence for all frequencies except main harmonics is smaller than 1 [Fig. 9(c)]. With further chaotization the level of coherence for other spectral components become lower but the coherence on the main frequency remain near 1 until temporal chaos is developed when the coherence on the main frequency is also decreased. Figure 10 demonstrates the dependence of the coherence function on the coupling value. The correspondent temporal regime was chosen as developed one-band chaotic attractor. Figures 10(a) and 10(b) correspond to space-periodic regimes. The coherence function is less than one for all frequencies except main peaks. Figures 10(c) and 10(d) relate to regimes with destroyed spatial structure. This case is characterized by value of coherence less than one on the main frequencies too. Comparing these figures with the spatio-temporal dynamics we see that spatial periodicity is connected with coherence on main peaks. Until the spectra of oscillations contain frequencies on which motions are fully coherent the chain preserves its averaged periodic spatial structure. If coherent function decreases for every frequency the spatial periodic structure is destroyed.

\section{Conclusion}

In the work we considered developing of the dynamics on the base of spatio-periodic phase structures in the chain of coupled period-doubling oscillators 
with periodic boundary conditions. These structures are traveling waves propagating along the chain with constant phase velocity. We have found that exact space-periodicity is preserved through period-doubling and tori birth bifurcations until transition to chaotic temporal behavior. Chaotic temporal behavior induces spatial chaos which remains spatio-periodic "on an average". The averaged spatio-periodic structure exists in the wide interval of the parameters and it is destroyed both with developing of chaos (at small coupling) and with decreasing of coupling (in the regime of oneband chaotic attractor). The presence of the averaged spatio-periodic structure is connected with the coherence of oscillations on main peaks in the mutual spectrum. It exists until the coherence function remains equal to 1 on base frequencies. We have built regions of stability for waves with different wavelengths and found that increasing of coupling leads to instability of short waves. In the work we describe typical bifurcations and present the map of main regimes originating from a simple traveling wave with changing parameters.

Evidently, considered particular spatio-periodic regimes and correspondent phase diagrams take place only for chains with the chosen length. However common regularities are found to exist also for arrays with other numbers of elements. We tested our results on chains with other lengths and obtained results are qualitatively similar to those described in this work (in our investigations we chose arrays up to 1024 elements length).

\section{Acknowledgments}

This material is based on work supported by the Naval Research Laboratory under Contract No. N68171-00-M-5430. The authors also thank the Civilian Research \& Development Foundation (Grant REC-006) and Russian Foundation of Basic Research (Grant 00-02-17512) for partial financial support.

\section{References}

Alexeyev, A. A., Osipov, G. V. \& Shalfeev, V. D. [1995] "Effects of square-wave modulation on CNN patterns," IEEE Trans. Circuits Syst. I: Fund. Th. Appl. 42(10), 700-704.

Anishchenko, V. S., Astakhov, V. V., Vadivasova, T. E., Sosnovtzeva, O. V., Wu, C. W. \& Chua, L. [1995]
"Dynamics of two coupled Chua's circuits," Int. J. Bifurcation and Chaos 5(6), 1677-1699.

Anishchenko, V. S., Astakhov, V. V., Nikolaev, V. V. \& Shabunin, A. V. [2000] "Chaotic synchronization in a network of symmetrically coupled oscillators," $J$. Commun. Technol. Electron. 45(2), 179-185.

Ashvin, P., Buescu, J. \& Stewart, I. [1994] "Bubbling of attractors and synchronization of chaotic oscillators," Phys. Lett. A193, 126-139.

Astakhov, V. V., Bezruchko, B. P., Gulyaev, Y. P. \& Seleznev, E. P. [1989] "Multistable states in dissipativelly coupled Feigenbaum systems," J. Techn. Phys. Lett. 15(3), 60-65.

Astakhov, V., Shabunin, A., Kapitaniak, T. \& Anishchenko, V. [1997a] "Loss of chaos synchronization through the sequence of bifurcations of saddle periodic orbits," Phys. Rev. Lett. 79, 1014-1017.

Astakhov, V. V., Shabunin, A. V., Silchenko, A. N., Strelkova, G. I. \& Anishchenko, V. S. [1997b] "Nonlinear dynamics of two coupled via capacity Chua's circuits," J. Commun. Technol. Electron. 42(3), 320-327.

Astakhov, V., Hasler, M., Kapitaniak, T., Shabunin, A. \& Anishchenko, V. [1998] "Effect of parameter mismatch on the mechanism of chaos synchronization loss in coupled systems," Phys. Rev. E58, 5620-5628.

Astakhov, V. V., Shabunin, A. V. \& Anishchenko, V. S. [1999] "Mechanisms of chaotic synchronization destruction in coupled cubic maps system," Appl. Nonlin. Dyn. 7(2), 3-11.

Belykh, I. V. \& Belykh, V. N. [2000] "Embedded manifolds and ordering of chaotic synchronization of diffusively coupled systems," Proc. Second Int. Conf. Control of Oscillations and Chaos, St. Petersburg, Russia, pp. 346-349.

Bonilla, L. L., Vicente, C. J. P. \& Spigler, R. [1998] "Time-periodic phases in populations of nonlinearly coupled oscillators with bimodal frequency distributions," Physica D113, 79-97.

Bressloff, P. C., Coombes, S. \& Souza, B. [1997] "Dynamics of a ring of pulse-coupled oscillators: Group theoretical approach," Phys. Rev. Lett. 79(15), 2791-2794.

Bressloff, P. C. \& Coombes, S. [1998] "Traveling waves in a chain of pulse-coupled oscillators," Phys. Rev. Lett. 80(21), 4815-4818.

Daido, H. [1996] "Onset of cooperative entrainment in limit-cycle oscillators with uniform all-to-all interactions: bifurcation of the order function," Physica D91, $24-66$.

Daido, N. [1997] "Strange waves in coupled-oscillator arrays: Mapping approach," Phys. Rev. Lett. 78(9), 1683-1684.

Fisher, D. S. [1985] "Sliding charge-density waves as a dynamic critical phenomenon," Phys. Rev. B31, 1396-1427. 
Fujisaka, H. \& Yamada, T. [1983] "Stability theory of synchronized motion in coupled-oscillator systems," Progr. Theor. Phys. 69, 32-47.

Fujigaki, H., Nishi, M. \& Shimada, T. [1996] "Synchronization of nonlinear systems with distinct parameters: Phase synchronization and metamorphosis," Phys. Rev. E53(4), 3192-3197.

Heagy, J. F., Carroll, T. L. \& Pecora, L. M. [1994] "Synchronous chaos in coupled oscillator systems," Phys. Rev. E50(3), 1874-1884.

Hu, G., Zhang, Y., Cerdeira, H. A. \& Chen, S. [2000] "From low-dimensional synchronous chaos to highdimensional desynchronous spatiotemporal chaos in coupled systems," Phys. Rev. Lett. 85(16), 3377-3380.

Huygens, C. [1673] Horoloqium Oscillatorium (Paris, France).

Inoue, M., Kawazoe, T., Nishi, Y. \& Nagadome, M. [1998] "Generalized synchronization and partial synchronization in coupled maps," Phys. Lett. A249, 69-74.

Kaneko, K. [1986] "Lyapunov analysis and information flow in coupled map lattices," Physica D23, 436-447.

Kaneko, K. [1989a] "Simulating physics with coupled map lattices," in Formation, Dynamics and Statistics of Patterns, Vol. 1 (World Scientific, Singapore), pp. $1-54$.

Kaneko, K. [1989b] "Towards thermodynamics of spatiotemporal chaos," Progr. Theor. Phys. 99, 263-287.

Komuro, M., Tokunaga, R., Matsumoto, T., Chua, L. O. \& Hotta, A. [1991] "Global bifurcation analysis of the double scroll circuit," Int. J. Bifurcation and Chaos 1(1), 139-182.

Kuramoto, Y. [1984] Chemical Oscillations. Waves and Turbulence (Springer, Berlin).

Kuramoto, Y. \& Nishikava, I. [1987] "Statistical macrodynamics of large dynamical system. Case of plane transition in oscillator communities," J. Stat. Phys. 49, 569-605.

Kuznetzov, A. P. \& Kuznetzov, S. P. [1990] "Spatial structures in dissipative media near the chaos threshold," Radiophysics 34(2), 142-146.

Lai, Y.-C., Grebogi, C., Yorke, J. A. \& Venkataramani, S. C. [1996] "Riddling bifurcation in chaotic dynamical systems," Phys. Rev. Lett. 77(1), 55-58.

Marino, I. P., Perez-Munuzuri, V., Perez-Villar, V., Sanchez, E. \& Matias, M. A. [2000] "Interaction of chaotic rotating waves in coupled rings of chaotic cells," Physica D128, 224-235.
Matias, M. A., Perez-Munuzuri, V., Marino, I. P., Lorenzo, M. N. \& Perez-Villar, V. [1997a] "Size instabilities in ring of chaotic synchronized systems," Europhys. Lett. 37(6), 379-384.

Matias, M. A., Guemez, J., Perez-Munuzuri, V., Marino, I. P., Lorenzo, M. N. \& Perez-Villar, V. [1997b] "Observation of a fast rotating wave in rings of coupled chaotic oscillators," Phys. Rev. Lett. 78(2), 219-222.

Matthews, P. C., Mirollo, R. E. \& Strogatz, S. H. [1990] "Dynamics of a large system of coupled nonlinear oscillators," Physica D52, 293-331.

Matthews, P. C. \& Strogatz, S. H. [1990] "Phase diagram for the collective behavior of limit-cycle oscillators," Phys. Rev. Lett. 65(14), 1701-1704.

Mirollo, R. E. \& Strogatz, S. H. [1990] "Synchronization of pulse-coupled biological oscillators," J. Appl. Math. 50, 1645-1662.

Mormann, F., Lehertz, K., David, P. \& Elger, C. E. [2000] "Mean phase coherence as a measure for phase synchronization and its application to the EEG of epilepsy patients," Physica D144, 358-369.

Pando, C. L. [2000] "Effects of a periodic perturbation on a discrete-time model of coupled oscillators," Phys. Lett. A273, 70-79.

Perez-Munuzuri, A., Perez-Munuzuri, V., Perez-Villar, V. \& Chua, L. O. [1993] "Spiral waves on 2-D array of nonlinear circuits," IEEE Trans. Circuits Syst. I: Fund. Th. Appl. 40(11), 872-877.

Rosenblum, M. G., Pikovsky, A. S. \& Kurths, J. [1996] "Phase synchronization of chaotic oscillations," Phys. Rev. Lett. 76(11), 1804-1807.

Rosenblum, M. G., Pikovsky, A. S. \& Kurths, J. [1997] "From phase to lag synchronization in coupled chaotic oscillators," Phys. Rev. Lett. 78, 4193-4196.

Rulkov, N. F., Sushchik, M., Tsimring, L. S. \& Abarbanel, H. D. I. [1995] "Generalized synchronization of chaos in directionally coupled chaotic systems," Phys. Rev. E51(2), 980-994.

Tass, P. [1997] "Phase and frequency shifts in a population of phase oscillators," Phys. Rev. E56(2), 2043-2060.

Strogatz, S. H. [2000] "From Kuramoto to Crawford: Exploring the onset of synchronization in population of coupled oscillators," Physica D143, 1-20.

Yeung, M. K. S. \& Strogatz, S. H. [1999] "Time delay model of coupled oscillators," Phys. Rev. Lett. 82(3), 648-651. 